Case report. A 50-year-old Swiss woman spent a number of weeks travelling around the UK, reporting to police that her family in Switzerland were working with their police, doctors and the mafia to kill her. She was therefore running away for her own safety, and trying to seek legal help to investigate. She had been in close contact with a woman in London who claimed to be able to give her legal aid in exchange for payment. On admission to the ward, it was felt that the legal aid was actually feeding her persecutory delusions.

Discussion. We discovered that she had in fact been detained in Switzerland prior to coming to the UK, and the discharge report was obtained which diagnosed her with Michael Kohlhaas syndrome and Folie a deux.

Conclusion. This poster will further explore Michael Kohlhaas syndrome and litigious paranoia, with connection to this case.

\section{Education and Training}

Trainees' perspective on the best use of supervision-hour in psychiatry training - a qualitative study

Raja Adnan Ahmed*, Mohamed Bader and Mohamed Flensham

Aneurin Bevan University Health Board

${ }^{\star}$ Corresponding author.

doi: 10.1192/bjo.2021.354

Aims. This study aims to identify the techniques to improve the quality of the weekly one to one supervision for Psychiatry trainees.

Method. An open-ended online questionnaire was prepared using principles of critical incident technique and distributed among psychiatry trainees working in various deaneries within the UK. The participants were asked to describe an example of a good and a bad supervision experience they had encountered during their training. In addition, participants were also requested to make suggestions to improve the supervision experience. All qualitative data were analysed using the thematic analysis approach, to identify common themes.

Result. A total of 53 trainees working in various deaneries across England and Wales, responded to the questionnaire. The respondents were at a different level of training in psychiatry from CT1-ST6 level. The supervision hour was reported to be useful for clinical case discussions, reflection on difficult cases and situations, pastoral support and wider issues relating to personal and professional development. Trainees appreciated a holistic scope for supervision rather than a narrow discussion of management of cases.

Trainees reported that the supervision hour should be trainee-led and tailored according to their unique learning needs. Participants also saw supervision hour as a safe space where they can receive constructive criticism and feedback on their performance. At times, trust and genuineness were appreciated, as well as the use of an informal tone by the supervisor. An effective supervision leads to trainees feeling valued.

Conclusion. Trainees acknowledged that the supervision hour is an effective tool in psychiatry training. Trainees should get regular, protected and uninterrupted time with consultants for weekly supervisions. Both trainees and trainers need to develop a better understanding of how this supervision experience could be improved and tailored to the individual learning needs of the trainee.
The person behind the label: co-production as a tool in teaching about borderline personality disorder

Nyakomi Adwok ${ }^{\star}$ and Sharon Nightingale

Leeds and York Partnership Foundation Trust

${ }^{*}$ Corresponding author.

doi: 10.1192/bjo.2021.355

Aims. The overarching aim of the session was to address and reduce stigma around Borderline Personality Disorder among doctors. The three main objectives were:

To increase empathy and understanding around Borderline Personality Disorder by exposing junior doctors to service user perspectives outside a clinical setting;

To address knowledge gaps identified by junior doctors in a self-reported questionnaire disseminated prior to the teaching session;

To offer junior doctors a basic psychological framework to base their assessment and formulation of service users with personality disorders.

Background. 'Borderline Personality Disorder: The Person Behind the Label' was the title of the first co-produced teaching session in the Leeds and York Partnership Foundation Trust (LYPFT). Prior to the teaching session, an online questionnaire was sent out to trainees. The results highlighted three key issues:

Negative attitudes towards service users with personality disorders;

Poor subjective knowledge of the psychological models of personality disorders;

Perception among trainees that they do not receive adequate training to deal with the challenges service users with personality disorders present.

Method. A teaching session was co-produced by a team of two service users, a principal clinical psychologist within the Leeds Personality Disorder Network (PDN) and a core Psychiatry trainee. It was delivered in a 75 minute session to 40 attendees consisting of both trainee doctors and consultants.

Result. Feedback was collected immediately after the session through the use of anonymous feedback forms. The response to the training was overwhelmingly positive with all 28 respondents rating the session as $4 / 5$ or $5 / 5$ on a satisfaction scale ranging from 1 (poor) to excellent (5). Key themes from the feedback included appreciation for the service user perspective and teaching on psychological theory. The fourth question in the questionnaire: "How will this teaching impact your work?" produced the highest number of responses $(25 / 28)$ and provided evidence that the above listed objectives of the session were met.

Conclusion. Co-produced teaching has great potential to address negative attitudes around highly stigmatised conditions by bridging the gap that often exists between service users and mental health professionals.

Provision of training for accommodation providers in the London Borough of Hackney - results of a scoping exercise and educational session pilot

Kate Aldersey ${ }^{\star}$, Sheraz Ahmad and Sian Mason

East London NHS Foundation Trust

${ }^{\star}$ Corresponding author.

doi: 10.1192/bjo.2021.356

Aims. To understand the level of training given to staff in providers of accommodation in the London Borough of Hackney across mental and physical health. 